\title{
Diffractive coupling in gold nanoparticle arrays and the effect of disorder
}

\author{
Baptiste Auguié* and William L. Barnes \\ School of Physics, University of Exeter, Stocker Road, Exeter, Devon, EX4 4QL, UK \\ *Corresponding author: ba208@exeter.ac.uk
}

Received November 6, 2008; accepted December 15, 2008;

posted January 9, 2009 (Doc. ID 103797); published February 4, 2009

\begin{abstract}
Two-dimensional arrays of gold nanoparticles with a periodicity commensurate with the wavelength of resonant excitation of localized plasmons have been shown to exhibit a strong long-range interaction between particles. We investigate experimentally the effect of varying the degree of disorder in the geometrical arrangement from a periodic to a disordered lattice with constant occupancy. We also investigate the effect of disorder arising from variations in particle size for a regular lattice, and the effect this has on the broadening of the spectral line shape is discussed. A coupled dipole model is used to describe the observed spectral features. (c) 2009 Optical Society of America

OCIS codes: $050.1950,050.5298,290.2200,290.5850,290.4210,290.3700$.
\end{abstract}

Collections of metallic nanoparticles display interesting optical properties due to the strong interaction of light with the particles that arises from the excitation of localized surface plasmon resonances (LSPRs) $[1,2]$. When the average particle separation is sufficiently small, light scattered by one such LSPRsupporting particle can significantly contribute to the excitation of its neighbors. Such coupling between particles can alter the single scattering response in a variety of ways depending on the distance between particles as well as their geometrical arrangement. Of particular interest here is the regime where the particles are separated by an average distance that is approximately equal to the wavelength of the light that propagates in the surrounding medium. In oneor two-dimensional ordered arrays of such particles, a delocalized surface mode can develop that couples together particles over large distances. Applications in nanoscale waveguides [3,4], sensing [5], and slow light $[4,6,7]$ have been envisaged. The case of twodimensional arrays of gold nanorods has recently received much interest as several experimental works [8-10] have confirmed theoretical predictions that had until now not been properly demonstrated. In particular, the extinction spectrum can display narrow features that result from a geometrical resonance associated with a coherent multiple scattering process. Here we investigate the influence of disorder in such structures on their spectral response by conducting experiments with a controlled distribution of particle positions and particle sizes.

We fabricated several gold nanoparticle arrays using electron-beam lithography on 25-mm-square 1-mm-thick fused silica substrates. This technique allows a precise control over the position and size of each individual particle. We made arrays with several distributions of particle positions as well as particle sizes as illustrated in Fig. 1. The spatial extent of the arrays was limited to $35 \mu \mathrm{m} \times 35 \mu \mathrm{m}$ to minimize the shape variation due to beam distortion at the edges of the available electron-beam write-field.

The particles (nominal size $120 \mathrm{~nm} \times 80 \mathrm{~nm}$ $\times 35 \mathrm{~nm}$ ) were deposited by thermal evaporation with a thin $(2 \mathrm{~nm})$ chromium layer being used to improve the adhesion of the gold onto the substrate. The particles were immersed in matching index fluid between two substrates so as to obtain an homogeneous refractive index environment $(n=1.46)$. The extinction spectra were obtained by measuring the transmittance at normal incidence with a collimated beam (divergence $<0.1^{\circ}$ ), using a $10 \times$ objective for the collection optics. A polarizer was used in the illumination path to selectively probe the LSPR associated with the long-axis of the nanoparticles.

Figure 2(a) presents the extinction spectra obtained from arrays that differed only in the degree of disorder of the particle positions. As it was noted in a previous study [9], the (nominally) ordered array presents a narrow extinction peak on the low-energy wing of the LSPR; this narrow feature occurs because of the coherent superposition of partial waves

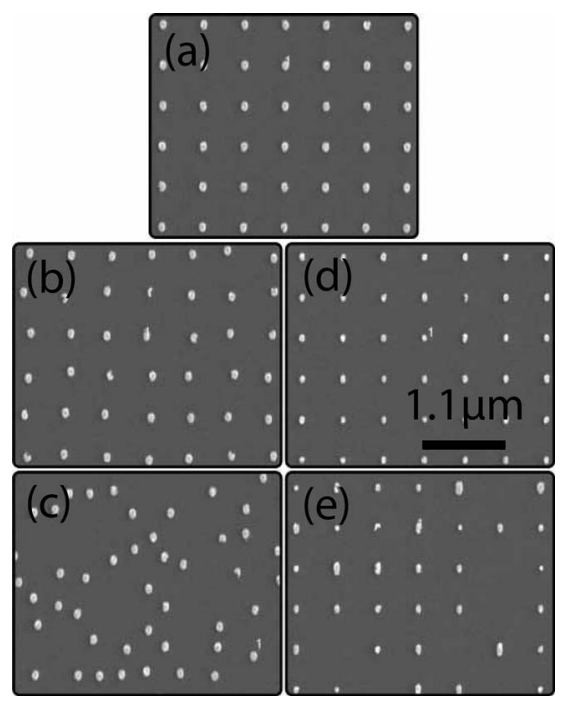

Fig. 1. Scanning electron micrographs of particle arrays. (a) Ordered array, i.e., no added disorder; (b) 10\% disorder in particle positions. (c) Pseudo-random positions; (d) $10 \%$ variation in the length of the long-axis of the particles; (e) $100 \%$ variation in the length of the long-axis of the particles. 


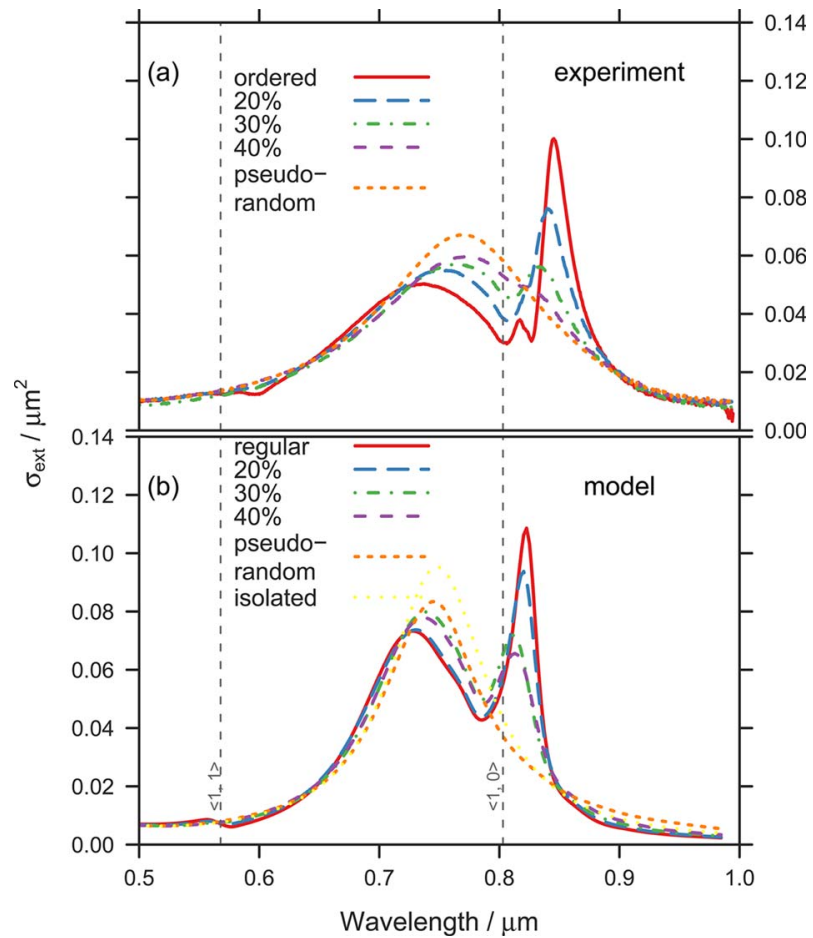

Fig. 2. (Color online) (a) Extinction spectra from five different arrays of nanoparticles of varying positional disorder but with constant average occupancy. The deviation from the ordered array is indicated in the legend as a percentage of the nominal regular particle separation $(550 \mathrm{~nm})$. The vertical dashed curves indicate the $\langle 1,0\rangle$ and $\langle 1,1\rangle$ diffraction edges for the periodic array in a homogeneous environment (refractive index 1.46). The nominal particle sizes were $120 \mathrm{~nm} \times 80 \mathrm{~nm} \times 35 \mathrm{~nm}$. (b) Calculated extinction spectra using a coupled dipole model with the same parameters.

scattered from all the particles in the array. We also observe that the extinction due to the excitation of the LSPR can be partially suppressed by the coherent scattering, which results in a dip in the extinction close to the diffraction edge. The strict periodicity is broken by displacing the particles from their regular locations in a random manner. As this disorder increases we note that the spectral shape evolves around the diffraction edge. In particular the sharp peak weakens and blueshifts in the disordered samples as the disorder increases, while the extinction due to the excitation of the LSPR regains its full strength. In the limit of spatially uncorrelated positions [labeled pseudorandom in Fig. 2(a)], we retrieve a smooth spectrum characteristic of an inhomogeneously broadened LSPR response. Our results are in good agreement with previous investigations on the effect of positional disorder in the optical response of metallic gratings (Nau et al. [11]). The design of each disordered configuration we studied was made so as to prevent overlap between particles as this would introduce an additional undesired distribution of particle sizes. The spatial pattern of the pseudorandom configuration was drawn from a Strauss point process $[12,13]$ that allows us to minimize the spatial correlation of the particles within the constraint of an exclusion zone around each particle.
Figure 2(b) is the result of a coupled dipole model with 441 dipoles arranged in a two-dimensional configuration to match that of the experiment. In this approximation, each particle is represented by a dipole of polarizability $\alpha$, the expression for which was chosen according to the study of Kuwata-Gonokami et al. [14] to describe accurately the influence of the particle size and shape and the relative permittivities of the metal and surrounding medium. This prescription includes terms to describe the dynamic depolarization and radiative damping that affect particles larger than the Rayleigh limit $(\ll \lambda)$. The interaction between dipoles is described by the dipolar field that participates in the excitation of the other dipoles [15]. The resulting system of coupled linear equations between the incident field $\left(E_{\text {inc }}\right)$ and the dipole moments $(P)$ is solved numerically, and the result is used to calculate the extinction cross section [15], $\sigma_{\text {ext }} \propto k \Im\left(E_{\text {i }}^{*} P\right)$. A good qualitative agreement is found between the modeling and the experimental results [compare Figs. 2(a) and 2(b)], confirming the interpretation of the narrow spectral feature as a result of coherent coupling between the particles mediated by dipolar radiation. The difference in intensity and width of the LSPR between the experiment and modeling can be explained by the approximations used in the model for the single particle response; additional damping mechanisms, such as surface roughness and the presence of the chromium underlayer, are not accounted for in the model. The small secondary peak seen in the experimental spectra between the two main resonances can be reproduced by allowing a small deviation from normal incidence (numerical results not shown). Furthermore, this simple model allows us to gain information on the single particle extinction spectrum that cannot be obtained from the experiment. This information is particularly useful in Fig. 3, where we consider an inhomogeneous distribution of particles. In Fig. 3(a) we present the effect of a dispersion of particle sizes on the optical spectrum of ordered arrays. Here it is seen that in contrast with the case of positional disorder the dip in the extinction curve associated with the diffraction edge never disappears. In addition, all spectral features broaden with an increasing dispersion in particle sizes. The LSPR peak is inhomogeneously broadened and redshifted by the wide distribution of aspect ratios and volumes of the different particles in the array. Consequently, a measurement of the plasmon lifetime in a collection of particles is often limited by this additional inhomogeneous broadening [16]. The width of the diffractive peak has, however, a different origin [3] not dictated by the lifetime of the LSPR but rather by factors such as array size, angular spread of illumination, and dispersion in the particle LSPR frequencies. It is the latter factor that we alter here by varying the particle sizes. To illustrate this point, we show in Fig. 3(b) the calculated extinction spectra for five normal distributions of disorder in particle sizes. Each curve is the average of 441 spectra obtained in the dipolar approximation with a range of long-axis lengths. No interaction between the particles is considered. The underlying distribu- 


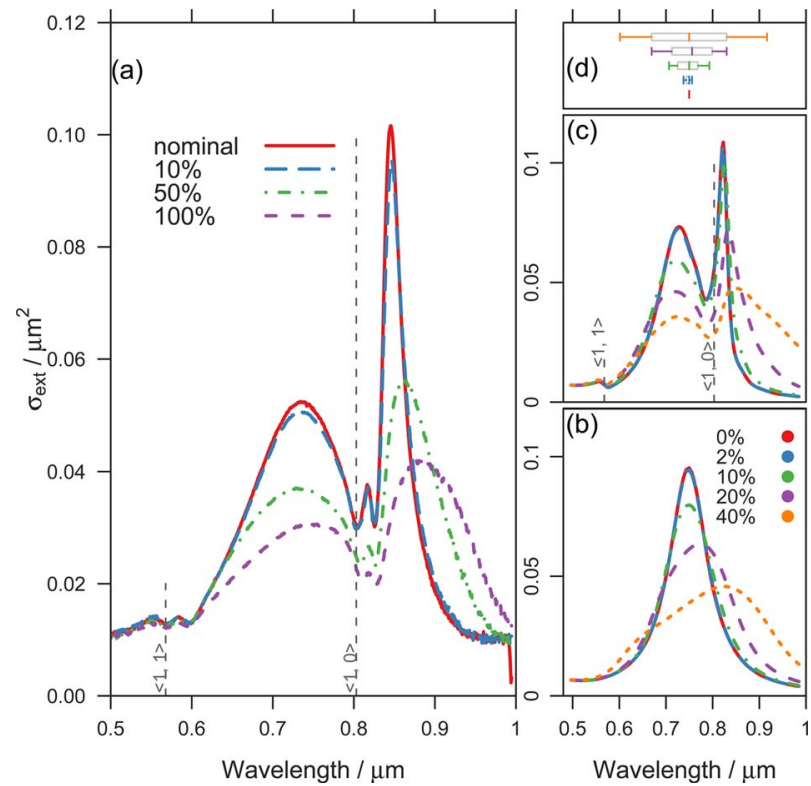

Fig. 3. (Color online) (a) Extinction spectra from arrays of nanoparticles with regular positions but a variation in particle sizes. The legend indicates the level of disorder introduced in the length of the long-axis of the nanorods. The vertical dashed curves indicate the $\langle 1,0\rangle$ and $\langle 1,1\rangle$ diffraction edges for the $550 \mathrm{~nm}$ periodic array in a 1.46 refractive index homogeneous environment. The nominal particle sizes were $120 \mathrm{~nm} \times 80 \mathrm{~nm} \times 35 \mathrm{~nm}$. (b) Calculated average spectra for five distributions of 441 ellipsoids. (c) Calculated spectra for a regular array of dipoles (pitch $550 \mathrm{~nm}$ ) using the distribution of individual LSPR frequencies shown in (d).

tion of the resonance frequencies of individual particles is displayed in Fig. 3(d). We see that a normal distribution of long-axis sizes results in a broadening of the overall spectrum and also introduces a redshift and skewness in the spectral shape. This can be understood by the nonlinear dependency of the LSPR spectrum on the length of the long-axis of the ellipsoids, in addition to the dispersion of the material permittivity that strengthens the intensity of the redshifted resonances. Figure 3(c) shows the calculated results for the same distributions of particle sizes but with the dipoles being part of an array of periodicity $550 \mathrm{~nm}$. The dipolar coupling results in a strong modification of the spectral line shape that qualitatively reproduces the experimental results. Introducing a distribution of particle sizes means that some particles will have LSPRs that no longer match well with the period; they thus contribute less effectively to the coherent coupling [9], and so by varying the distribution of particle sizes, fewer particles support a LSPR that can contribute to the coherent coupling.
This Letter provides some insight into the coherent interaction between particles ordered in a periodic two-dimensional array. In particular we have shown that the periodic arrangement results in a sharp spectral feature near the diffraction edge that can be totally suppressed by altering the spatial correlation between the scattering centers. Of much importance for potential applications is the width of the diffractive peak, and we assessed the influence of a dispersion in particle sizes on the intensity and width of the spectral line shape. We used a coupled dipole model to disentangle the effects of inhomogeneous broadening and coherent multiple scattering.

The authors would like to thank Vincent Loke for sharing his implementation of the discrete dipole approximation from which the coupled dipole model used in this work was inspired and the Engineering and Physical Sciences Research Council (EPSRC) (EP/C52389X/1) for funding through the Basic Technology project "2D Attogram Surface Plasmon Resonance Imaging." W. L. Barnes thanks the Royal Society for support through a Wolfson Merit Award.

\section{References}

1. U. Kreibig and L. Genzel, Surf. Sci. 156, 678 (1985).

2. S. Link and M. El-Sayed, Int. Rev. Phys. Chem. 19, 409 (2000).

3. V. A. Markel and A. Sarychev, Phys. Rev. B 75, 085426 (2007)

4. S. A. Maier, P. G. Kik, H. A. Atwater, S. Meltzer, E. Harel, B. E. Koel, and A. A. Requicha, Nature Mater. 2, 229 (2003).

5. A. Grigorenko, H. Gleeson, Y. Zhang, N. Roberts, A. Sidorov, and A. Panteleev, Appl. Phys. Lett. 88, 124103 (2006)

6. Z. V. Vardeny and A. Nahata, Nat. Photonics 2, 75 (2008).

7. A. A. Govyadinov and V. A. Markel, Phys. Rev. B 78, 035403 (2008).

8. V. G. Kravets, F. Schedin, and A. N. Grigorenko, Phys. Rev. Lett. 101, 087403 (2008).

9. B. Auguié and W. L. Barnes, Phys. Rev. Lett. 101, 143902 (2008).

10. Y. Chu, E. Schonbrun, T. Yang, and K. B. Crozier, Appl. Phys. Lett. 93, 181108 (2008).

11. D. Nau, A. Schoenhardt, C. Bauer, A. Christ, T. Zentgraf, J. Kuhl, M. W. Klein, and H. Giessen, Phys. Rev. Lett. 98, 133902 (2007).

12. A. Baddeley and R. Turner, J. Stat. Software 12, 1 (2005).

13. D. Strauss, Biometrika 62, 467 (1975).

14. H. Kuwata-Gonokami, H. Tamaru, K. Esumi, and K. Miyano, Appl. Phys. Lett. 83, 4625 (2003).

15. G. C. Schatz and R. P. Van Duyne, J. Chem. Phys. 103, 869 (1995).

16. C. Sönnichsen, T. Franzl, T. Wilk, G. von Plessen, J. Feldmann, O. Wilson, and P. Mulvaney, Phys. Rev. Lett. 88, 077402 (2002). 